\title{
Role of Intestinal Microflora on Necrotizing Enterocolitis in Preterm Infants
}

\author{
Lijuan Wu ${ }^{1}$ Hung-Chih Lin ${ }^{2,3}$ \\ ${ }^{1}$ Clinical Laboratory, Bao'an Maternity and Child Health Hospital, \\ Shenzhen, Guangdong Province, China \\ ${ }^{2}$ Children's Hospital of China Medical University, Taichung, Taiwan \\ ${ }^{3}$ School of Chinese Medicine, China Medical University, \\ Taichung, Taiwan \\ J Pediatr Biochem 2015;5:55-59.
}

\begin{abstract}
Address for correspondence Hung-Chih Lin, MD, No. 2 Yuh Der Road, Taichung 404, Taiwan (e-mail: d0373.cmuh@gmail.com).
\end{abstract}

\begin{abstract}
Keywords

- intestinal microflora

- necrotizing enterocolitis

- preterm infants

Necrotizing enterocolitis (NEC) remains a devastating intestinal disease in preterm very low-birth-weight (VLBW: $<1,500 \mathrm{~g}$ ) infants. Though the pathogenesis of NEC in preterm VLBW infants is not fully explored, numerous clinical evidence and laboratory data have supported that the bacterial colonization is a critical factor for NEC development. Recent evidence has suggested that NEC is associated with both unusual intestinal microbial species and an overall reduction in the diversity of microbiota. Nonetheless, preterm infants have very unique microflora colonization in the intestinal tract because many preterm infants are born via cesarean section and are mandated to develop intestinal microflora colorization within the complex neonatal intensive care unit. Furthermore, preterm infants show delayed colonization by "healthy commensal" organisms, especially bifidobacteria and lactobacilli. All these data suggest that low colonization of Bifidobacterium and Lactobacillus in preterm VLBW infants may serve as a predisposing factor in microbial infection and NEC. Based on these findings, researchers have tried to use probiotics to prevent NEC in prematurity; there are 27 randomized controlled trials and 7 recent meta-analyses which enrolled a total of 6,655 preterm infants. All the evidence confirmed that oral probiotics effectively prevent NEC and death in preterm infants. Premature rat model and updated meta-analysis further showed that combined probiotics strains resulted in a marked reduction of the incidence of NEC. Future study should focus on the head-to-head control trial on different probiotics regimen and possible use of the microbiome of breast-fed preterm infants for total fecal transplantation to eradicate NEC.
\end{abstract}

\section{Introduction}

Despite overall decreased mortality and morbidity in preterm very low-birth-weight (VLBW: $<1,500 \mathrm{~g}$ ) infants over the past decades, necrotizing enterocolitis (NEC), a devastating emergency intestinal disease in preterm VLBW infants, remains 5 to $6 \%$ and even increase despite much effort on peri-postnatal

received

July 24, 2015 accepted after revision

August 4, 2015 published online

October 6, 2015
Issue Theme Intestinal Microbiota of Childhood: Dysbiosis and Diseases; Guest Editors: Yuichiro Yamashiro, MD, PhD, and Kazunari Kaneko, MD, PhD care. ${ }^{1}$ More worrisome, approximately 20 to $30 \%$ of VLBW infants with NEC will die $^{2}$ and the remaining survivors will be at substantial risk for long-term complications, including impaired neurodevelopment and short bowel syndrome. ${ }^{3}$ Around $10 \%$ of infants with NEC are term or near-term neonates; the risk factors of NEC in term neonates include congenital heart diseases, prenatal asphyxia, and patent
Copyright $\odot 2015$ by Georg Thieme Verlag KG, Stuttgart · New York
DOI http://dx.doi.org/ 10.1055/s-0035-1564575. ISSN 1879-5390. 
ductus arteriosus requiring indomethacin therapy. ${ }^{4}$ Thus, pathophysiology of NEC should be different between term and preterm infants. We thus review the role of intestinal microflora on NEC in preterm infants only.

The pathogenesis of NEC in preterm infants is multifactorial; the immaturity of the gastrointestinal (GI) tract in terms of motility, digestive function, circulatory regulation, barrier function, and immune defense are the important underlying risk factors. The widely accepted hypothesis at present is that enteral feeding (providing substrate) in the presence of intestinal colonization by pathogens provokes an inappropriately accentuated inflammatory response by immature intestinal epithelial cells of preterm neonates; these result in a disruption of the intestinal barrier and translocation of bacterial endotoxins. ${ }^{5,6}$

\section{Unique Features of the Preterm Intestinal Microbiome}

Intestinal microfloras are composed of approximately $10^{14}$ bacteria, which represent approximately 10 times the number of cells in the human body.

Upon delivery, the neonate is exposed to microbes from a variety of sources, including maternal vaginal, fecal, and skin bacteria. Initial colonization of the infant gut is highly influenced by the mother's vaginal and fecal bacterial communities, which include facultative anaerobes such as streptococci and Enterobacteriaceae. Indeed, the first and most important phase of normal colonization occurs when the newborn fetus passes through the birth canal and ingests maternal vaginal and colonic microorganisms. These bacteria further proliferate when oral feeding is initiated. After 48 hours of birth, the number of bacteria is already as high as approximately $10^{4}$ to $10^{6}$ colony-forming units per milliliter of intestinal contents. Many factors may influence this process, including gestational age (preterm or full term), mode of delivery (vaginal or cesarean section), infant diet (breastfeeding or formula), birth environment of neonatal intensive care unit (NICU), and use of drugs such as antibiotics or proton pump inhibitors. ${ }^{7}$

Preterm infants are very unique in regard to sudden interruption of the in utero environment; many preterm infants are born via cesarean section and could not have own maternal nipple feeding. This makes preterm infants nearly no chance to get maternal generic microflora colorization in the GI tract. Furthermore, preterm infants are mandated to develop within the NICU and therefore they acquire their GI tract microbiota within the confines of the NICU where colonization is significantly influenced by iatrogenic manipulations such as the frequent administrations of diverse drugs (broad-spectrum antibiotics, opioids, and histamine-2 receptor antagonists) or numerous instrumentations (endotracheal tubes, feeding tubes, and suctioning tubes).

An environment is not only the factor that affects the microflora colorization of the GI tract in preterm infants. Studies have demonstrated that immature intestinal epitheliums are decreased in intestinal motility, and are character- ized by the alterations of surface glycoconjugate glycosylation patterns, intestinal mucus densities, and carbohydrate contents: All of these characteristics may alter bacterial binding. ${ }^{8,9}$ It is reasonable inference that interactions between the immaturity of the GI tract and specific environmental factors of NICU alter the preterm infant's endogenous microbiota and its susceptibility to NEC.

On the other hand, it is also suggested that genetic factors play an important role on microflora colorization of the GI tract in infants. Studies have demonstrated that related preterm twins share similar gut microbiome development, even within the complex environment of NICU. This finding appears to be a result of shared genetic and immunomodulatory factors as well as exposures to the same maternal microbiome during birth, the same skin contact, and the same expressed breast milk. ${ }^{10}$ However, many very preterm infants are born via caesarean section and could not have own maternal nipple feeding. This suggests that shared factors (genetic and environmental) are important in determining the gut microbiome even in an environment with many complex variable factors that also affect microbial community development. ${ }^{11,12}$

\section{Intestinal Microbial Ecology Affecting Necrotizing Enterocolitis}

Almost four decades ago, Sántulli et al observed that bacterial colonization was a critical factor in NEC pathogenesis in early descriptions of the disease. ${ }^{13}$ After that, numerous clinical evidence and laboratory data supporting this assertion have accumulated. These include the following: changes in the microbial community have been reported to be associated with NEC and sepsis in unrelated preterm neonates ${ }^{14,15}$; specific pathogens caused numerous NEC outbreaks ${ }^{16}$; gut colonization in preterm infants is often delayed ${ }^{17}$; abnormal gut microbiota such as decreased diversity was found particularly in infants who developed NEC. ${ }^{18-20}$

Studies reporting specific bacterial associations with NEC suggest a role for Proteobacteria, commonly Enterobacteriaceae. $^{14,21}$ Other evidence supporting the role of gut microbiota is that an early antibiotic exposure can be a risk factor for NEC because of its effect on gut microbial colonization. ${ }^{22,23}$ In fact, more recently, investigators have utilized culture-independent assays in piglet NEC models and showed that NEC is associated with disturbances in the intestinal microbiota. 24,25

Recent study by Torrazza et al further disclosed that infants who develop NEC did exhibit a different pattern of microbial colonization compared with controls as following ${ }^{26}$ : there was a higher proportion of Proteobacteria (61\%) 2 weeks and of Actinobacteria (3\%) 1 week before diagnosis of NEC compared with controls (19 and $0.4 \%$, respectively); it was characterized by the lower numbers of Bifidobacteria counts and Bacteroidetes proportions in the weeks before NEC diagnosis; in the first fecal samples obtained during week 1 of life, a novel signature sequence, distinct from but matching closest to Klebsiella pneumoniae, that was strongly associated with NEC development later in life, was detected. 
Meanwhile, three recent studies by $16 \mathrm{~S}$ rRNA gene analysis have identified an abundance of organisms from the phylum Proteobacteria (which includes many commonly observed gram-negative pathogens) in fecal samples of babies with NEC. ${ }^{14,15,18}$ Additional findings included a loss of gut microbial diversity ${ }^{18}$ and depletion of enterococcal populations in NEC samples. ${ }^{15}$

Because fecal microbes do not necessarily correlate with the organisms found within intestinal mucosa, studying microbes within intestinal tissue removed from preterm babies might provide more information of the cross talk between microflora and epithelium in the pathogenesis of NEC. In this regard, a study of microbes present in formalinfixed intestinal samples resected from 24 infants with NEC demonstrated an abundance of organisms from the phylum Proteobacteria and the genus Ralstonia. Interestingly, a significant correlation was found between the clinical finding of pneumatosis intestinalis and the presence of clostridial species (Clostridium butyricum and Clostridium paraputrificum). ${ }^{27}$

These studies suggest that NEC is associated with both unusual intestinal microbial species and an overall reduction in the diversity of microbiota. ${ }^{14,18,28}$ Taken together, the variability of these results suggests that NEC may not result from a single causative species but more likely from a currently undefined dysbiosis. Further evidence also comes from the findings that NEC was not observed in germ-free animals ${ }^{29}$ and NEC did not occur in the intrauterine environment, despite ingestion of $150 \mathrm{~mL} / \mathrm{kg} /$ day of amniotic fluid that contains proteins, carbohydrates, fat, immunoglobulin, and electrolytes. ${ }^{30}$ All of these findings strongly suggest that bacterial colonization is an important factor in the pathogenesis of this disease.

New evidence disclosed that the majority of the differentially abundant genes in the NEC patient were associated with carbohydrate metabolism and mapped to members of the family Enterobacteriaceae. ${ }^{31}$ This may indicate an adaptation of the community to an altered profile of substrate availability for specific members as a first step toward the development of NEC.

Although dysbiosis of the gut microbiome is implicated in disease development, no single causative agent has been convincingly identified yet as a biomarker of NEC. This is because the data in this regard are limited in extremely lowbirth-weight infants who carry the highest risk for NEC. The association between gut microbiome and development of NEC needs further investigation to provide detailed pathophysiology and most logic approach to prevent NEC in vulnerable infants.

\section{Clinical Observations and Experimental Models Support the Critical Role of Microbiome in Necrotizing Enterocolitis}

Numerous experimental models and clinical observations have confirmed the critical role of gut microbiota in NEC, for example, preterm neonates show delayed colonization by "healthy commensal" organisms, especially bifidobac- teria and lactobacilli. ${ }^{32-34}$ These data suggest that low colonization of Bifidobacterium and Lactobacillus in VLBW infants may serve as a predisposing factor in microbial infection and NEC. Based on these findings, it is speculated that probiotics could be potentially used to prevent NEC in prematurity.

Using animal model, Caplan et $\mathrm{al}^{35}$ and Butel et $\mathrm{al}^{36}$ showed that bifidobacterial supplement in rat and quail model resulted in intestinal colonization and subsequent reduction in NEC-like lesion. Researcher began to examine whether probiotics could prevent the occurrence of NEC. Kitajima et al started the first trial of early administration of Bifidobacterium breve to preterm infants, but failed to prove the hypothesis because of inadequate sample size. ${ }^{37}$

Subsequently, Lactobacillus acidophilus ${ }^{38}$ and Bifidobacterium infantis ${ }^{39}$ had been used as probiotics to reduce the incidence of NEC in Italy and Columbia, respectively. They reported the contradicting results, that is, the former was negative, ${ }^{38}$ while the latter was positive. ${ }^{39}$ Meanwhile, our randomized controlled trials either with single or multicenter center was the first to prove that administration of probiotics that contain Lactobacillus and Bifidobacterium in VLBW infants reduces the incidence and severity of NEC. ${ }^{40,41}$

After our study, there were 25 randomized controlled trials and 7 recent meta-analyses that enrolled a total of 6,655 preterm infants and confirmed that oral probiotics effectively prevent NEC and death ${ }^{42-48}$ : the use of different probiotics in all studies has made the selection of an optimal probiotic regimen difficult, and therefore, clinicians are facing challenges in assessing which probiotics are most effective to prevent NEC in preterm VLBW infants.

Our premature rat model showed that administration of a mixture of probiotic strains with Bifidobacterium bifidum and Bifidobacterium longum was most effective in preventing death and NEC in this animal model ${ }^{49}$; an updated meta-analysis showed that combined probiotics strains resulted in a marked reduction in the incidence of NEC with a pooled OR of 0.37 and improved mortality with a pooled OR of 0.58 in preterm infants ( $\leq 34$ weeks' gestation) or those of a birth weight $\leq 1,500 \mathrm{~g}$ (Chang HY et al, 218 Congress of Taiwan Pediatrics Society 2015 April; Taipei, Taiwan: accepted by the European Clinical Nutrition 2015).

Though there is no clinical randomized controlled trial examining the protective effect of $B$. longum subspecies infantis (B. infantis) on NEC, $B$. infantis is unique in its prodigious capacity to digest and consume any human milk oligosaccharide structure. It is also characterized that $B$. infantis grows better than other bacterial strains in the presence of human milk oligosaccharides, and displays anti-inflammatory activity in premature intestinal cells, and decreases intestinal permeability. ${ }^{50}$ In addition, a large repertoire of bacterial genes encoding an array of glycosidases and oligosaccharide transporters are not found in other bacterial species. Further study is needed to investigate the protective effect on NEC of single B. infantis compared with combined probiotics or synbiotics. 


\section{Future Direction}

We believe that identification of simple species may not be a robust enough measure to identify the links between NEC and gut microbes. The advanced deep sequencing by the currently available sequencing platforms makes it possible to characterize the potentially important organisms that are present at a low abundance. Available data suggest that the colonization pattern of the healthy full-term infants who are breast fed may represent optimal colonization for the preterm infant as well. Future study should focus on the head-to-head control trial on different probiotics regimen and possible use of the microbiome of breast-fed preterm infants for total fecal transplantation to eradicate NEC.

\section{References}

1 Neu J, Walker WA. Necrotizing enterocolitis. N Engl J Med 2011; 364(3):255-264

2 Fitzgibbons SC, Ching Y, Yu D, et al. Mortality of necrotizing enterocolitis expressed by birth weight categories. J Pediatr Surg 2009;44(6):1072-1075, discussion 1075-1076

3 Hintz SR, Kendrick DE, Stoll BJ, et al; NICHD Neonatal Research Network. Neurodevelopmental and growth outcomes of extremely low birth weight infants after necrotizing enterocolitis. Pediatrics 2005;115(3):696-703

4 Bolisetty S, Lui K, Oei J, Wojtulewicz J. A regional study of underlying congenital diseases in term neonates with necrotizing enterocolitis. Acta Paediatr 2000;89(10):1226-1230

5 Hsueh W, Caplan MS, Qu XW, Tan XD, De Plaen IG, Gonzalez-Crussi F. Neonatal necrotizing enterocolitis: clinical considerations and pathogenetic concepts. Pediatr Dev Pathol 2003;6(1):6-23

6 Chen AC, Chung MY, Chang JH, Lin HC. Pathogenesis implication for necrotizing enterocolitis prevention in preterm very-lowbirth-weight infants. J Pediatr Gastroenterol Nutr 2014;58(1): 7-11

7 Johnson CL, Versalovic J. The human microbiome and its potential importance to pediatrics. Pediatrics 2012;129(5):950-960

8 Snyder JD, Walker WA. Structure and function of intestinal mucin: developmental aspects. Int Arch Allergy Appl Immunol 1987; 82(3-4):351-356

9 Dai D, Nanthakumar NN, Savidge TC, Newburg DS, Walker WA. Region-specific ontogeny of alpha-2,6-sialyltransferase during normal and cortisone-induced maturation in mouse intestine. Am J Physiol Gastrointest Liver Physiol 2002;282(3): G480-G490

10 Stewart CJ, Marrs EC, Nelson A, et al. Development of the preterm gut microbiome in twins at risk of necrotising enterocolitis and sepsis. PLoS ONE 2013;8(8):e73465

11 Palmer C, Bik EM, DiGiulio DB, Relman DA, Brown PO. Development of the human infant intestinal microbiota. PLoS Biol 2007;5(7):e177

12 Hamady M, Lozupone C, Knight R. Fast UniFrac: facilitating highthroughput phylogenetic analyses of microbial communities including analysis of pyrosequencing and PhyloChip data. ISME J 2010;4(1):17-27

13 Sántulli TV, Schullinger JN, Heird WC, et al. Acute necrotizing enterocolitis in infancy: a review of 64 cases. Pediatrics 1975; 55(3):376-387

14 Mai V, Young CM, Ukhanova M, et al. Fecal microbiota in premature infants prior to necrotizing enterocolitis. PLoS ONE 2011; 6(6):e20647

15 Stewart CJ, Marrs EC, Magorrian S, et al. The preterm gut microbiota: changes associated with necrotizing enterocolitis and infection. Acta Paediatr 2012;101(11):1121-1127
16 Boccia D, Stolfi I, Lana S, Moro ML. Nosocomial necrotising enterocolitis outbreaks: epidemiology and control measures. Eur J Pediatr 2001;160(6):385-391

17 Blakey JL, Lubitz L, Barnes GL, Bishop RF, Campbell NT, Gillam GL. Development of gut colonisation in pre-term neonates. J Med Microbiol 1982;15(4):519-529

18 Wang Y, Hoenig JD, Malin KJ, et al. 16S rRNA gene-based analysis of fecal microbiota from preterm infants with and without necrotizing enterocolitis. ISME J 2009;3(8):944-954

19 Mshvildadze M, Neu J, Shuster J, Theriaque D, Li N, Mai V. Intestinal microbial ecology in premature infants assessed with non-culture-based techniques. J Pediatr 2010;156(1):20-25

20 Cilieborg MS, Boye M, Sangild PT. Bacterial colonization and gut development in preterm neonates. Early Hum Dev 2012;88 (Suppl 1):S41-S49

21 Carlisle EM, Poroyko V, Caplan MS, Alverdy JA, Liu D. Gram negative bacteria are associated with the early stages of necrotizing enterocolitis. PLoS ONE 2011;6(3):e18084

22 Cotton CM. Early, prolonged use of postnatal antibiotics increased the risk of necrotising enterocolitis. Arch Dis Child Educ Pract Ed 2010;95(3):94

23 Kuppala VS, Meinzen-Derr J, Morrow AL, Schibler KR. Prolonged initial empirical antibiotic treatment is associated with adverse outcomes in premature infants. J Pediatr 2011;159(5):720-725

24 Bjornvad CR, Thymann T, Deutz NE, et al. Enteral feeding induces dietdependent mucosal dysfunction, bacterial proliferation, and necrotizing enterocolitis in preterm pigs on parenteral nutrition. Am J Physiol Gastrointest Liver Physiol 2008;295(5):G1092-G1103

25 Cilieborg MS, Boye M, Mølbak L, Thymann T, Sangild PT. Preterm birth and necrotizing enterocolitis alter gut colonization in pigs. Pediatr Res 2011;69(1):10-16

26 Torrazza RM, Ukhanova M, Wang X, et al. Intestinal microbial ecology and environmental factors affecting necrotizing enterocolitis. PLoS ONE 2013;8(12):e83304

27 Smith B, Bodé S, Petersen BL, et al. Community analysis of bacteria colonizing intestinal tissue of neonates with necrotizing enterocolitis. BMC Microbiol 2011;11:73

28 Morrow AL, Lagomarcino AJ, Schibler KR, et al. Early microbial and metabolomic signatures predict later onset of necrotizing enterocolitis in preterm infants. Microbiome 2013;1(1):13

29 Morowitz MJ, Poroyko V, Caplan M, Alverdy J, Liu DC. Redefining the role of intestinal microbes in the pathogenesis of necrotizing enterocolitis. Pediatrics 2010;125(4):777-785

30 Davies PA. Low birthweight infants: immediate feeding recalled. Arch Dis Child 1991;66(4):551-553

31 Claud EC, Keegan KP, Brulc JM, et al. Bacterial community structure and functional contributions to emergence of health or necrotizing enterocolitis in preterm infants. Microbiome 2013;1(1):20

32 Gewolb IH, Schwalbe RS, Taciak VL, Harrison TS, Panigrahi P. Stool microflora in extremely low birthweight infants. Arch Dis Child Fetal Neonatal Ed 1999;80(3):F167-F173

33 Schwiertz A, Gruhl B, Löbnitz M, Michel P, Radke M, Blaut M. Development of the intestinal bacterial composition in hospitalized preterm infants in comparison with breast-fed, full-term infants. Pediatr Res 2003;54(3):393-399

34 Jacquot A, Neveu D, Aujoulat F, et al. Dynamics and clinical evolution of bacterial gut microflora in extremely premature patients. J Pediatr 2011;158(3):390-396

35 Caplan MS, Miller-Catchpole R, Kaup S, et al. Bifidobacterial supplementation reduces the incidence of necrotizing enterocolitis in a neonatal rat model. Gastroenterology 1999;117(3):577-583

36 Butel MJ, Roland N, Hibert A, et al. Clostridial pathogenicity in experimental necrotising enterocolitis in gnotobiotic quails and protective role of bifidobacteria. J Med Microbiol 1998;47(5): 391-399

37 Kitajima H, Sumida Y, Tanaka R, Yuki N, Takayama H, Fujimura M. Early administration of Bifidobacterium breve to preterm infants: 
randomised controlled trial. Arch Dis Child Fetal Neonatal Ed 1997;76(2):F101-F107

38 Dani C, Biadaioli R, Bertini G, Martelli E, Rubaltelli FF. Probiotics feeding in prevention of urinary tract infection, bacterial sepsis and necrotizing enterocolitis in preterm infants. A prospective double-blind study. Biol Neonate 2002;82(2):103-108

39 Hoyos AB. Reduced incidence of necrotizing enterocolitis associated with enteral administration of Lactobacillus acidophilus and Bifidobacterium infantis to neonates in an intensive care unit. Int J Infect Dis 1999;3(4):197-202

$40 \mathrm{Lin} \mathrm{HC}$, Su BH, Chen AC, et al. Oral probiotics reduce the incidence and severity of necrotizing enterocolitis in very low birth weight infants. Pediatrics 2005;115(1):1-4

41 Lin $\mathrm{HC}$, Hsu CH, Chen HL, et al. Oral probiotics prevent necrotizing enterocolitis in very low birth weight preterm infants: a multicenter, randomized, controlled trial. Pediatrics 2008;122(4):693-700

42 Barclay AR, Stenson B, Simpson JH, Weaver LT, Wilson DC. Probiotics for necrotizing enterocolitis: a systematic review. J Pediatr Gastroenterol Nutr 2007;45(5):569-576

43 Deshpande G, Rao S, Patole S. Probiotics for prevention of necrotising enterocolitis in preterm neonates with very low birthweight: a systematic review of randomised controlled trials. Lancet 2007; 369(9573):1614-1620
44 Alfaleh K, Anabrees J, Bassler D. Probiotics reduce the risk of necrotizing enterocolitis in preterm infants: a meta-analysis. Neonatology 2010;97(2):93-99

45 Deshpande G, Rao S, Patole S, Bulsara M. Updated meta-analysis of probiotics for preventing necrotizing enterocolitis in preterm neonates. Pediatrics 2010;125(5):921-930

46 Wang $\mathrm{Q}$ Dong J, Zhu Y. Probiotic supplement reduces risk of necrotizing enterocolitis and mortality in preterm very low-birthweight infants: an updated meta-analysis of 20 randomized, controlled trials. J Pediatr Surg 2012;47(1):241-248

47 AlFaleh K, Anabrees J, Bassler D, Al-Kharfi T. Probiotics for prevention of necrotizing enterocolitis in preterm infants. Cochrane Database Syst Rev 2014;4:CD005496

48 Yang Y, Guo Y, Kan Q Zhou XG, Zhou XY, Li Y. A meta-analysis of probiotics for preventing necrotizing enterocolitis in preterm neonates. Braz J Med Biol Res 2014;47(9):804-810

49 Wu SF, Chiu HY, Chen AC, Lin HY, Lin HC, Caplan M. Efficacy of different probiotic combinations on death and necrotizing enterocolitis in a premature rat model.J Pediatr Gastroenterol Nutr 2013; 57(1):23-28

50 Underwood MA, German JB, Lebrilla CB, Mills DA. Bifidobacterium longum subspecies infantis: champion colonizer of the infant gut. Pediatr Res 2015;77(1-2):229-235 